\title{
Temperature Dependence of Ferroelectric Mode of Frequency, Dielectric Constant and Loss Tangent in Deuterated Triglycine Sulphate
}

\author{
NAVEEN KOHLI and TRILOK CHANDRA UPADHYAY
}

Physics Department, H N B Garhwal University (A Central University), Srinagar, Garhwal, U K 246174, India

kohlinav1001@gmail.com

Received 20 November 2015 / Accepted 10 December 2015

\begin{abstract}
The model values of physical properties for deuterated triglycine sulphate crystal in theoretically derived expressions for FE mode frequency, dielectric constant and loss tangent in dependence of these quantities have been calculated and compared with experimental data of literature value. A good agreement is found.
\end{abstract}

Keywords: Ferroelectrics, Anharmonics, Dielectric, Loss tangent, Phase transition, Isotope effect

\section{Introduction}

Triglycine sulphate $\mathrm{NH}_{2} \mathrm{CH}_{2}(\mathrm{COO})_{3} \mathrm{H}_{2} \mathrm{SO}_{4}$ (TGS) undergoes ferroelectric transition at $49{ }^{\circ} \mathrm{C}$. On deuteration, the transition temperature shifts to $60{ }^{\circ} \mathrm{C}$, showing large isotope effect. This fact suggests that hydrogen bond plays an important role in phase transition mechanism. The ferroelectric structure is monoclinic with space group $\mathrm{P} 2_{1} / \mathrm{m}$ and the lattice dimensions are a $=9.15 \AA, b=12.69 \AA, c=\AA, \beta=105.4^{\circ}$. This is low temperature phase. The paraelectric phase is monoclinic with space group $14 / \mathrm{m}$.

Extensive experimental studies on TGS crystal and its deuterated form (DTGS) have been carried out by many experimentalists. Batra and $\mathrm{Lal}^{1}$ have done crystal growth study. Electrical and mechanical properties have been studied by Murludharan et al., ${ }^{2}$. Dielectric measurements were carried out on TGS and DTGS by Alexandru et al., ${ }^{3}$. Vibrational spectral studies of pure and doped DTGS crystal were done by Arun et $a l^{4}$. Zolfagharien and Dizazi ${ }^{5}$ have made growth and characterization study of DTGS. Prasalov et al., ${ }^{6}$ have done hysteresis study of DTGS crystal. Khanum and Podder ${ }^{7}$ have done crystallization and characterization of TGS crystal doped with $\mathrm{NiSO}_{4}$. Shreekumar and Philip ${ }^{8}$ have done ultrasonic study of DTGS crystal. Aravazhi et al. ${ }^{9}$ have done dielectric and loss tangent measurements on DTGS crystal. Theoretical studies on TGS crystal were initiated by Blinc et al., ${ }^{10}$ who used Ising model. Chaudhuri et al., ${ }^{11}$ have considered a two-sub lattice pseudo spin model. They used Green's function method and 
obtained soft mode frequency, susceptibility, dielectric constant and transition temperature. These authors ${ }^{11}$ have not considered order phonon anharmonic interaction. They have decoupled the correlation at any stage. So that they could not produce better and convincing results. In the earlier paper one of authors ${ }^{12}$ they have considered phonon anharmonic interactions terms ${ }^{13}$.

In the present study we shall fit model values of physical quantities in the expressions obtained in one of authors earlier paper ${ }^{12}$ for deuterated TGS crystal. Temperature dependences of ferroelectric mode frequency, dielectric constant and loss tangents will be calculated for DTGS crystal. Theoretical results will be compared with experimental results of Aravazhi et al .

\section{Calculation and Results}

In our earlier paper a two-sub lattice pseudo spin lattice coupled mode model along with third and fourth order phonon anharmonic interactions terms has been considered for TGS crystal. The Green's function is differentiated twice with respect to time $t$ and $t^{\prime}$ with the help of modified Hamiltonian which is then set into Dyson's equation form. As a result Green's function was obtained as;

$$
G(\omega)=\pi^{-1} \Omega\left\langle S_{i 1}^{x}\right\rangle \delta_{i j}\left[\left(\omega^{2}-\hat{\Omega}^{2}\right)+2 i \Omega \Gamma(\omega)\right]^{-1},
$$

where $\hat{\Omega}^{2}=\widetilde{\Omega}^{2}+\Delta(\omega)$,

$$
\begin{aligned}
\widetilde{\Omega}^{2} & =a^{2}+b^{2}-b c \\
a & =2 J_{0}\left\langle S_{1}^{z}\right\rangle+K_{0}\left\langle S_{2}^{z}\right\rangle, \\
b & =2 \Omega \\
c & =2 J_{0}\left\langle S_{1}^{z}\right\rangle+K_{0}\left\langle S_{2}^{x}\right\rangle
\end{aligned}
$$

$\Delta(\omega)$ and $\Gamma(\omega)$ are shifts and width of response function of Green's function $G(\omega)$. Expression of $\Delta(\omega)$ and $\Gamma(\omega)$ have been given in our earlier paper ${ }^{12}$. Solving Eq (2) the FE mode frequency was obtained as;

$$
\hat{\Omega}^{2}=\frac{1}{2}\left\{\left(\tilde{\widetilde{\omega}}_{k}^{2}+\tilde{\widetilde{\Omega}}\right) \pm\left[\left(\tilde{\widetilde{\omega}}_{k}^{2}-\tilde{\Omega}^{2}\right)^{2}+16 V_{i k}\left\langle S_{1}^{x}\right\rangle \Omega\right]\right\} .
$$

The dielectric constant $\varepsilon$ is related to susceptibility $\chi$ as $\varepsilon=4 \pi \chi$ which in turn related $^{18}$ to retarded Green's function (2) as $\chi=-2 N \mu^{2} G(\omega+i X)$. Therefore putting value of Green's function (1) we have obtained value of dielectric constant $\mathcal{E}$ as;

$$
\varepsilon=\left(-8 \pi N \mu^{2}\right)\left\langle S^{x}\right\rangle\left(\omega^{2}-\tilde{\Omega}\right)\left[\left(\omega^{2}-\hat{\Omega}\right)^{2}+4 \Omega^{2} \Gamma^{2}\right]^{-1} .
$$

The dissipation of power when crystal is exposed to electromagnetic field is expressed as loss tangent. The loss tangent is the ratio of imaginary to real parts of dielectric constant,

$$
\tan \delta=\frac{2 \Omega \Gamma(\omega)}{\hat{\Omega}^{2}}
$$


By using model values of physical quantities for deuterated TGS crystal given in Table 1, temperature dependence of ferroelectric mode frequency, dielectric constant and loss tangent have been calculated and plotted in graph (Figures 1-3). Calculated temperature dependences have been compared with experimental values of Aravazhi et al., ${ }^{9}$.

Table 1. Model values of physical quantities of deuterated TGS crystal

\begin{tabular}{cccccccc}
\hline $\mathrm{T}_{\mathrm{c}},{ }^{\circ} \mathrm{C}$ & $\Omega \mathrm{cm}^{-1}$ & $\mathrm{~J}_{0} \mathrm{~cm}^{-1}$ & $\overline{\mathrm{v}} \mathrm{cm}^{-1}$ & $\omega_{\mathrm{k}} \mathrm{cm}^{-1}$ & $\mathrm{~A}_{\mathrm{k}} \mathrm{cm}^{-1}$ & $\mathrm{C},{ }^{\circ} \mathrm{C}$ & $\mathrm{N} \mu, \mu \mathrm{C} / \mathrm{cm}^{2}$ \\
\hline 60.70 & 0.01 & 470 & 15 & 1.92 & 14.22 & 4600 & $2.30\left(\right.$ at $\left.30.70{ }^{\circ} \mathrm{C}\right)$ \\
\hline
\end{tabular}

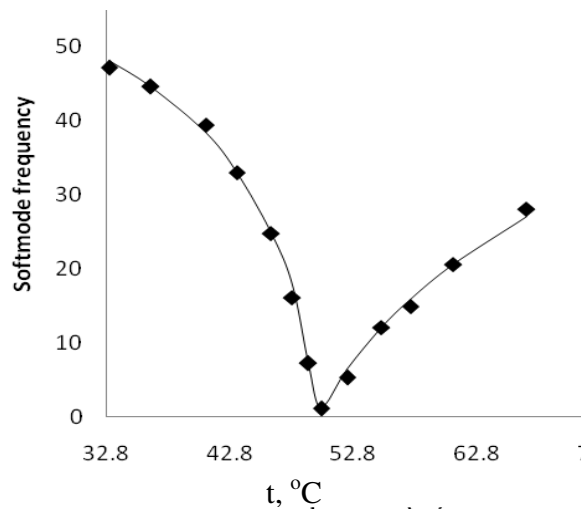

Figure 1. Calculated temperature dependence of soft mode frequency of DTGS crystal-, correlated Exp ${ }^{9}$ values

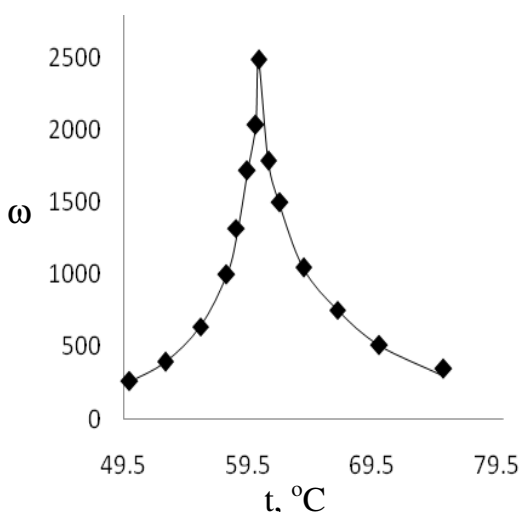

Figure 2. Calculated temperature dependence of dielectric constant in DTGS crystal-, $\operatorname{Exp}^{9} \bullet$

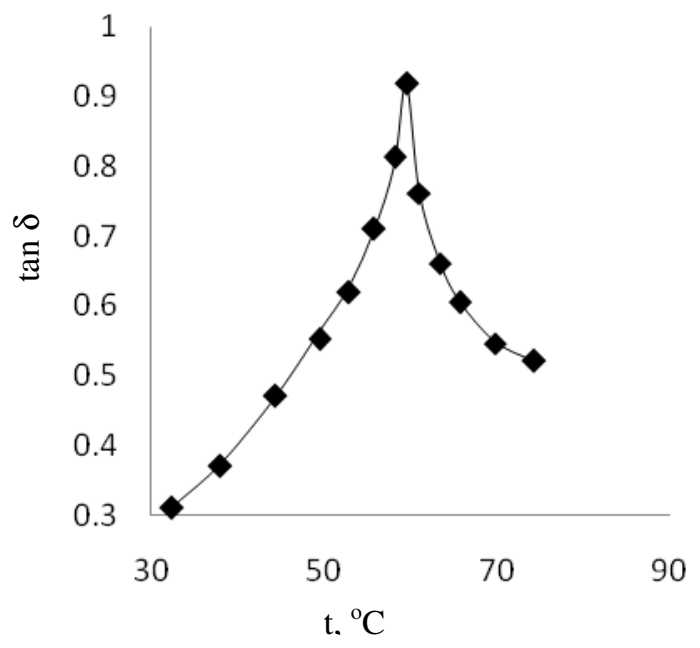

Figure 3. Calculated temperature dependence of loss tangent in DTGS crystal- $\operatorname{Exp}^{9}$

\section{Discussion}

In the present work the temperature dependences of ferroelectric frequency, dielectric constant and loss tangent have been calculated. Theoretically calculated results compare with experimentally reported results of Aravazhi et al., for deuterated TGS very well. 
The main aim of the present work is to explain isotope effect in DTGS crystal. On deuteration, the transition temperature shifts from $49^{\circ}$ to $60{ }^{\circ} \mathrm{C}$ and both dielectric constant and loss tangent versus temperature curves shift from to new values. Our expression for transition temperature $T_{c}$ with value for DTGS explains small isotope effect on $T_{c}$. Our expression (8), (9) and (10) explain the temperature dependences of ferroelectric mode frequency, dielectric constant and loss tangent for DTGS crystal. The Change in tunneling frequency is mainly responsible for isotope effect in DTGS crystal. Hence with the expressions obtained for DTGS crystal with different values of physical quantities, for DTGS Crystal, one can explain ferroelectric and isotope effect in deuterated TGS crystal.

\section{Conclusion}

Present study reveals that the two sub lattice pseudo spin-lattice coupled mode mode model along with third and fourth order phonon anharmonic interactions terms explain well the ferroelectric and dielectric behaviors of deuterated TGS crystal. Theoretical results agree with experimental results of Aravazhi et al., ${ }^{9}$ which shows the applicability of the present model.

\section{Acknowledgement}

Authors are grateful to Eminent Physicist Prof B S Semwal (Former Head) for his encouragement and to Prof Vinay Gupta (Delhi Univ.), Prof U C Naithani, Prof S C Bhatt and Dr Manish Uniyal for their encouragements. They are thankful to Dr Sandeep Sharma and Dr Ashish Nautiyal for the Discussions.

\section{References}

1. Lal and Batra A K, Ferroelectrics, 1993, 142(1), 51-82; DOI:10.1080/00150199308237884

2. Murlidharan R, Mohankumar R, Dhanasekaran R, Tripathi A K, Jayavel R, Ramasamy P, Mater Lett., 2003, 57(21), 3291-3295; DOI:10.1016/S0167$577 \mathrm{X}(03) 00050-8$

3. Alexandru H V and Ciceron A B, Ferroelectrics, 1997, 202(1), 173-182; DOI:10.1080/00150199708213474

4. Arun K J, Batra A K, Aggarwal M D, Almuatasim Alomari, Am J Mater Sci., 2015, 5, 3A, 48-54, DOI:105923/s.materials.201502.08

5. Zolfagharien N and Rezagholipour Dizaji H, Indian J Pure Appl Phys., 2015, 53, 234-238.

6. Prasalov B N, Palagin M Y and Gorbatenko V V, Ferroelectrics, 1998, 214(1), 325328; DOI:10.1080/00150199808220272

7. Khanum F, Podder J, J Crystal Process and Technol., 2011, 1(3), 49-54, DOI: $10.4236 /$ jept.2011.13008

8. Shreekumar R and Philip J, Ferroelectrics, 1994, 160(1), 23-33; DOI:10.1080/00150199408007691

9. Aravazhi S, Jayavel R and Subramanian C, Ferroelectrics, 1997, 200(1), 279-286; DOI:10.1080/00150199708008612

10. Blinc R, Detoni S and Pintar M, Phys Rev., 1961, 124, 1036; DOI:10.1103/PhysRev.124.1036

11. Chaudhuri B K, Chaudhary K R and Banerjee S, Phy Rev B, 1988, 38, 689; DOI:10.1103/PhysRevB.38.689

12. Upadhyay T C and Sandeep Sharma, Asian J Chem., 2011, 23(12), 5629-5631

13. Semwal B S and Sharma P K, Prog Theor Phys (Jpn), 1974, 51(3), 639-655; DOI:10.1143/PTP.51.639

14. Zubarev D N, Sov Phys Usp., 1960, 3, 320. 